\title{
Biomechanical Analysis of a Pedicle Screw-Rod System with a Novel Cross-Link Configuration
}

\author{
Yasuhiro Nakajima ${ }^{1}$, Masahito Hara ${ }^{1}$, Daisuke Umebayashi ${ }^{1}$, Shoichi Haimoto ${ }^{2}$, \\ Yu Yamamoto ${ }^{1}$, Yusuke Nishimura ${ }^{2}$, Toshihiko Wakabayashi ${ }^{2}$ \\ ${ }^{1}$ Department of Neurosurgery, Inazawa Municipal Hospital, Inazawa, Japan \\ ${ }^{2}$ Department of Neurosurgery, Nagoya University Graduate School of Medicine, Nagoya, Japan
}

Study Design: The strength effects of a pedicle screw-rod system supplemented with a novel cross-link configuration were biomechanically evaluated in porcine spines.

Purpose: To assess the biomechanical differences between a conventional cross-link pedicle screw-rod system versus a novel crosslink instrumentation, and to determine the effect of the cross-links.

Overview of Literature: Transverse cross-link systems affect torsional rigidity, but are thought to have little impact on the sagittal motion of spinal constructs. We tested the strength effects in pullout and flexion-compression tests of novel cross-link pedicle screw constructs using porcine thoracic and lumbar vertebrae.

Methods: Five matched thoracic and lumbar vertebral segments from 15 porcine spines were instrumented with 5.0-mm pedicle screws, which were then connected with 6.0-mm rods after partial corpectomy in the middle vertebral body. The forces required for construct failure in pullout and flexion-compression tests were examined in a randomized manner for three different cross-link configurations: un-cross-link control, conventional cross-link, and cross-link passing through the base of the spinous process. Statistical comparisons of strength data were analyzed using Student's $t$-tests.

Results: The spinous process group required a significantly greater pullout force for construct failure than the control group ( $p=0.036)$. No difference was found between the control and cross-link groups, or the cross-link and spinous process groups in pullout testing. In flexion-compression testing, the spinous processes group required significantly greater forces for construct failure than the control and cross-link groups ( $p<0.001$ and $p=0.003$, respectively). However, there was no difference between the control and cross-link groups.

Conclusions: A novel cross-link configuration that features cross-link devices passing through the base of the spinous processes increased the mechanical resistance in pullout and flexion-compression testing compared to un-cross-link constructs. This configuration provided more resistance to middle-column damage under flexion-compression testing than conventional cross-link configuration.

Keywords: Cross-link; Spinous process; Biomechanics; Spinal fusion; Pedicle screw-rod

\section{Introduction}

The mechanical strength provided by instrumentation is very important for the clinical success of spinal fusion. Various available pedicle screw-rod systems provide adequate strength. Importantly, the torsional stiffness of

Received Feb 1, 2016; Revised Apr 5, 2016; Accepted Apr 8, 2016

Corresponding author: Yasuhiro Nakajima

Department of Neurosurgery, Inazawa Municipal Hospital, 100 Numa, Nazuka-cho, Inazawa City, Aichi 492-8510, Japan

Tel: +81-587-32-2111, Fax: +81-587-32-2151, E-mail: y-nakajima@city.inazawa.aichi.jp 
pedicle screw-rod systems is augmented using cross-link devices [1]. Although cross-links increase lateral bending stiffness, their effects are predominately observed during torsional loading [2]. Some studies have indicated that increasing the number of cross-links within a construct provides increases resistance to lateral bending and torsional loading [3-5]. However, despite advances in cross-link instrumentation, spine surgeons experience instrument failure in long fusion for thoracic and thoracolumbar deformities.

Several common adverse outcomes are observed after thoracolumbar long fusion, including adjacent segment degeneration, proximal and distal junctional kyphosis usually across the thoracolumbar junction, and instrument failure. Adjacent segment disease occurs more frequently with extended long fusion (18.5\%) than short fusion (5.2\%) [6]. Although proximal kyphosis can occur at a higher incidence $(0 \%-46 \%)$ [7] than distal or caudal kyphosis (maximum, 28\%) [8], distal kyphosis may require more extensive surgery [8]. In addition, sublaminar wires, hooks, and hook-pedicle screw hybrid constructs are often used at the proximal or distal end of posterior instrumentation, but they cannot effectively prevent instrument failure [9].

We believe that cross-links in an " $\mathrm{X}$ " configuration would improve torsional stiffness relative to transverse cross-link systems. We hypothesized that a novel crosslink configuration, with cross-link devices passing through the base of the spinous process, would increase mechanical resistance against pullout and flexion loads compared to conventional cross-link constructs.

\section{Materials and Methods}

\section{Pullout testing}

Fifteen juvenile female porcine thoracic spines, weighing 35 to $40 \mathrm{~kg}$, were harvested from the most caudal five thoracic vertebrae. Muscle and soft tissue from each rib was removed, and the rib cage was removed while keeping the facet joint capsules and spinal ligaments intact. Specimens were frozen at $-20^{\circ} \mathrm{C}$ until testing and defrosted just before testing. Test segments were instrumented by a single spine surgeon using bilaterally placed $5.0 \times 35-\mathrm{mm}$ pedicle screws (Easy Spine, LDR Medical, Troyes, France). The screw entry point was selected in accordance with established principles [10] with minor modifications to accommodate the thoracic levels of the porcine vertebral anatomy. Of all available large animal models, porcine specimens have been found to most closely reflect normal human anatomy, with pedicle dimensions suitable for instrumentation with the screws selected $[11,12]$.

Pedicle screws were inserted after cannulating the pedicles under direct visualization. The screws were tapped to the pedicle-body junction with a tap $1 \mathrm{~mm}$ smaller than the screw diameter, and $6.0-\mathrm{mm}$ rods were connected bilaterally at all segments, except for the middle corpectomy segment. Each screw was inserted so that the normal spinal curvature of the specimen was not altered by the attachment of the longitudinal rods. Minor rotational adjustments were made to incorporate the rods. Three crosslink configurations were tested, as shown in Fig. 1: uncross-link controls $(n=5)$, conventional cross-links $(n=5)$, and cross-links passing through the base of the spinous processes $(n=5)$. Two 2 -mm cross-link devices were applied in each construct. The vertebral specimens were mounted in a custom-designed fixture that constrained the vertebral body and was properly oriented for axial pullout loading. For pullout testing, the specimens were aligned such that the pedicles were in line with the crosshead loading axis. The specimens were then gripped and pulled unaxially at the rod between the two terminal pedicle screws using an AG-X testing machine (Shimadzu Inc., Kyoto, Japan) with a $10 \mathrm{kN}$ load cell at a rate of 2 $\mathrm{mm} / \mathrm{min}$ (Fig. 2). The tests were stopped when the force

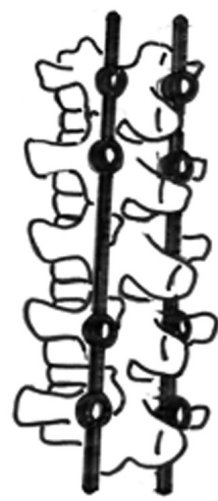

CONT (A)

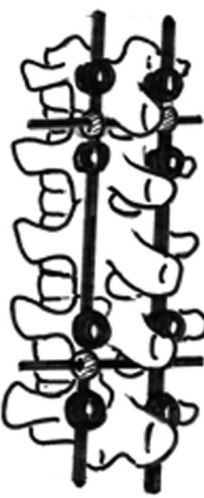

$\mathrm{CL}$

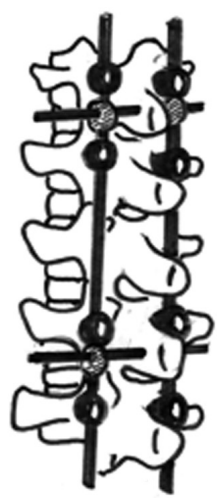

$S P$
Fig. 1. Schematic illustrating the cross-link configurations evaluated in this study. (A) Un-cross-link control (CONT). (B) Conventional crosslinks (CL). (C) Cross-links passing through the base of the spinous processes (SP). 

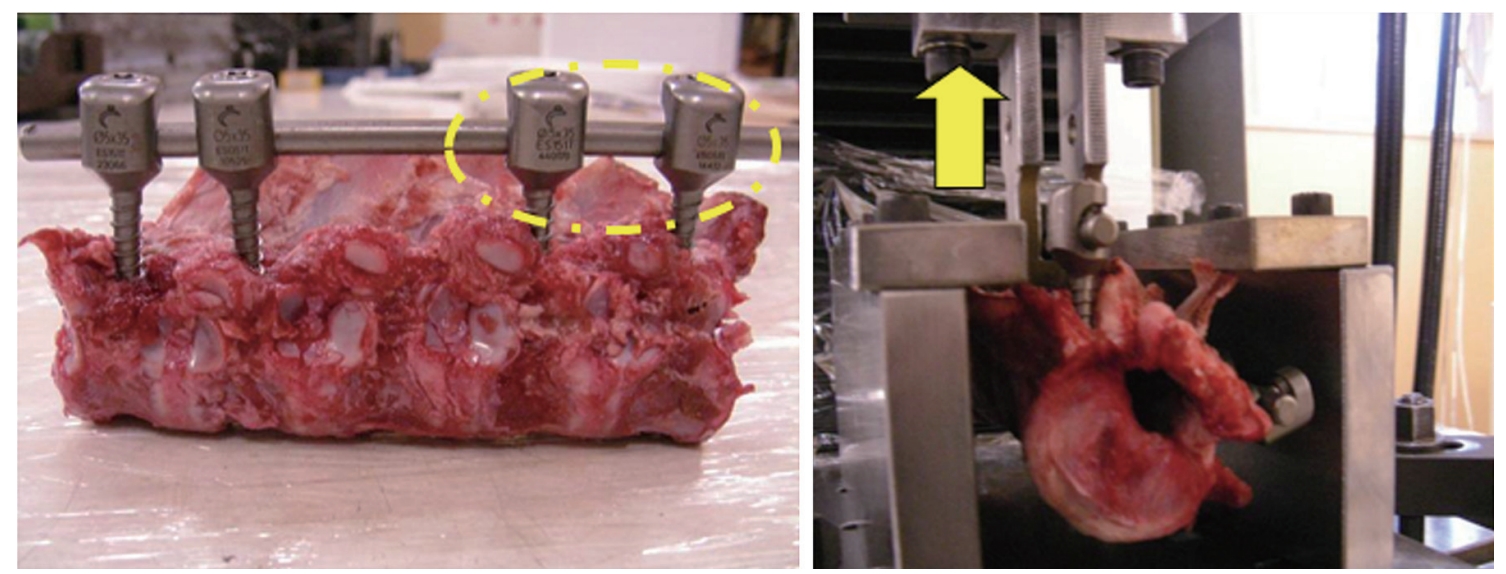

Fig. 2. The pedicle screw-rod system was gripped and pulled unaxially at the rod between the two terminal pedicle screws using an $A G-X$ testing machine.

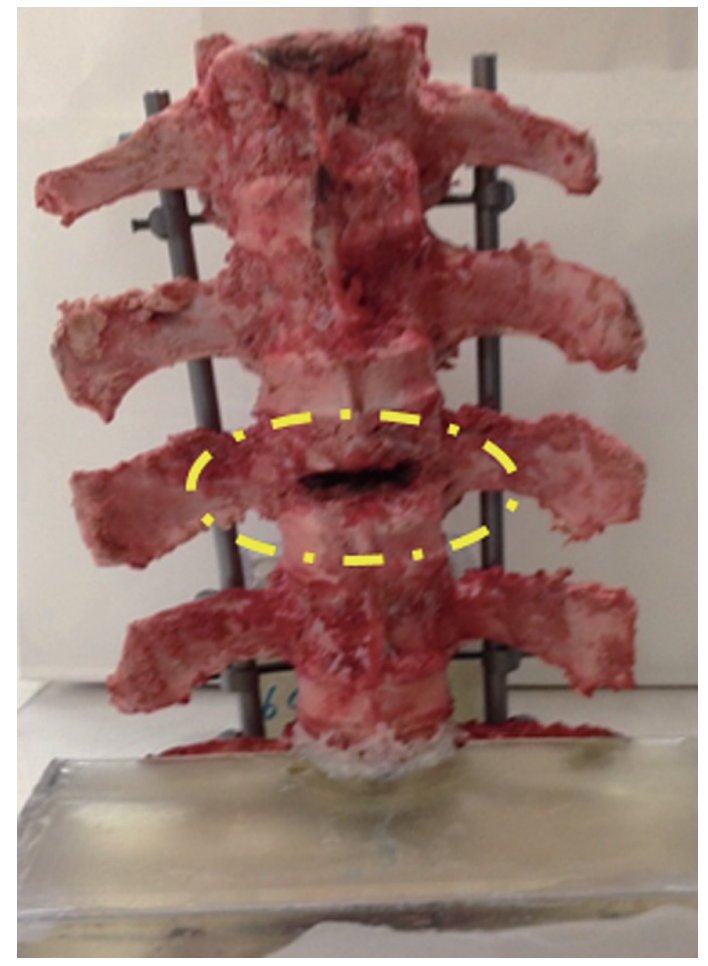

Fig. 3. A partial corpectomy in the ventral aspect of the middle vertebral body of an instrumented lumbar spine.

was reduced by $>20 \%$ or bone fracture occurred. The highest load value was recorded as the pullout load.

\section{Flexion-compression testing}

Fifteen porcine lumbar spines were harvested from the most caudal five lumbar vertebrae for axial-compression testing. The test segments were instrumented using bilater- ally placed $5.0 \times 35-\mathrm{mm}$ pedicle screws and $6.0-\mathrm{mm}$ connective rods. The three cross-link configurations were divided in the same manner as pullout testing. A partial corpectomy with a vertical and horizontal size of $10 \times 30 \mathrm{~mm}$ and a depth of $10 \mathrm{~mm}$ was made in the ventral aspect of the middle vertebral body (Fig. 3). This model became unstable and increased transfer of flexion loads to the instrumentation, reducing the stabilizing effects of the vertebral column. Hart et al. [13] found a statistically significant increase in stiffness among models with segmental pedicle screws compared to non-segmental pedicle screws, and observed the highest difference between cross-link nonsegmental and segmental models for 5-vertebrae fusion models in axial rotation and flexion testing.

The cranial and caudal vertebral body of each segment was embedded in a custom-designed jig that constrained the vertebral body and properly oriented the segment for axial compression loading. For flexion-compression testing, the cranial endplate of the specimens was compressed using the surface of a rectangular platform of the aforementioned AG-X testing machine (Shimadzu Inc.) with a $10 \mathrm{kN}$ load cell at a rate of $1 \mathrm{~mm} / \mathrm{min}$ (Fig. 4). The tests were stopped when the force was reduced by $>20 \%$ or fracture of the dorsal wall of the middle vertebral body with a partial corpectomy occurred. The highest load value was recorded as the flexion load.

Comparisons of load for each of the three constructs were made using Student's $t$ tests for the pullout and axial-compression tests. Statistical significance was set at $p<0.05$. Statistical analyses were performed using standard software (StatView for Windows ver. 5; SAS Institute Inc, Cary, NC, USA). 

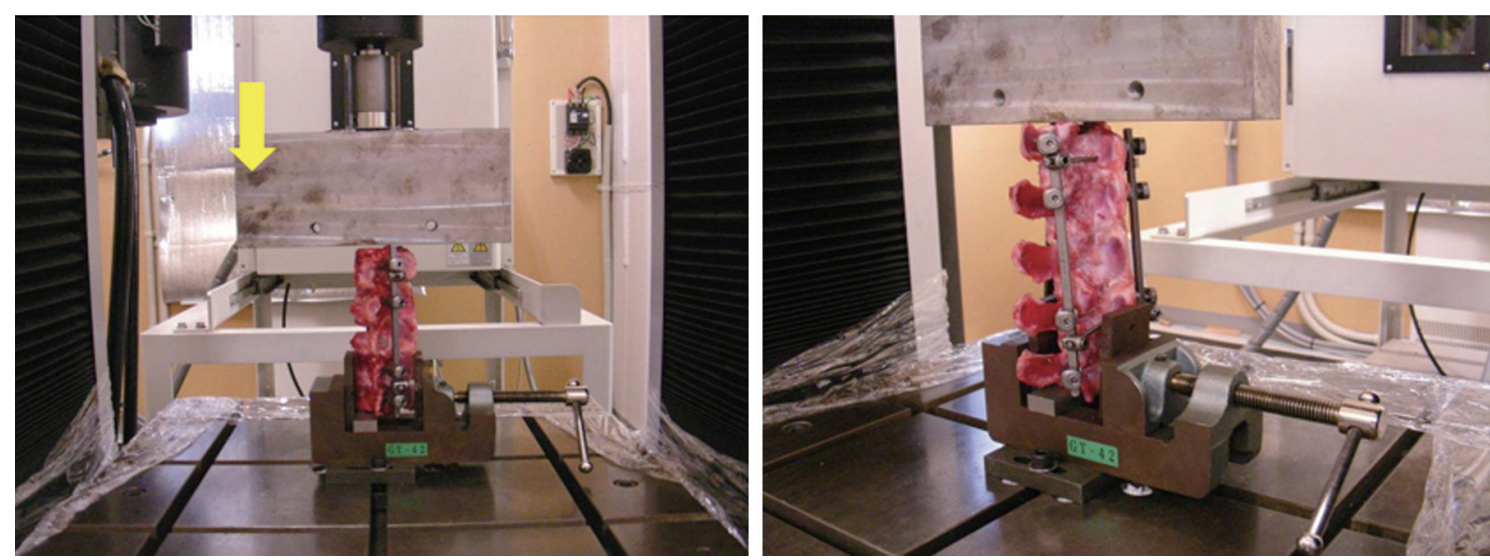

Fig. 4. The instrumented lumbar spine was axially compressed by using the surface of a rectangular platform of an AG-X testing machine.

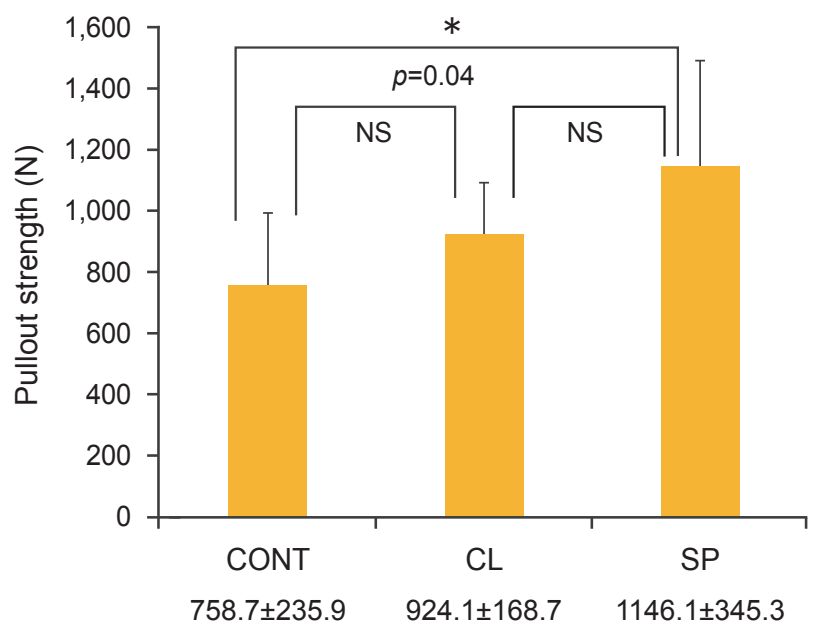

Fig. 5. The improved cross-link construct (SP) was significantly stronger than the un-cross-link controls during pullout testing of the pedicle screw-rod system. No significant differences were detected among the control (CONT) and conventional cross-link configurations (CL), and among the $\mathrm{CL}$ and the improved cross-link construct (SP). SP, cross-link passing through the base of the spinous process; NS, not significant. ${ }^{*} p<0.05$.

\section{Results}

During pullout testing, the spinous process group demonstrated a significantly greater pullout load than the control group ( $p=0.036)$. There was no statistical difference between the control and cross-link ( $p=0.12$ ), or cross-link and spinous processes $(p=0.12)$ groups (Table 1, Fig. 5). During flexion-compression testing, the spinous processes group demonstrated a significantly increased flexion load with respect to the other two configurations tested (control, $p<0.001$; cross-link, $p=0.003$ ). However, no difference was detected between the control and cross-link groups

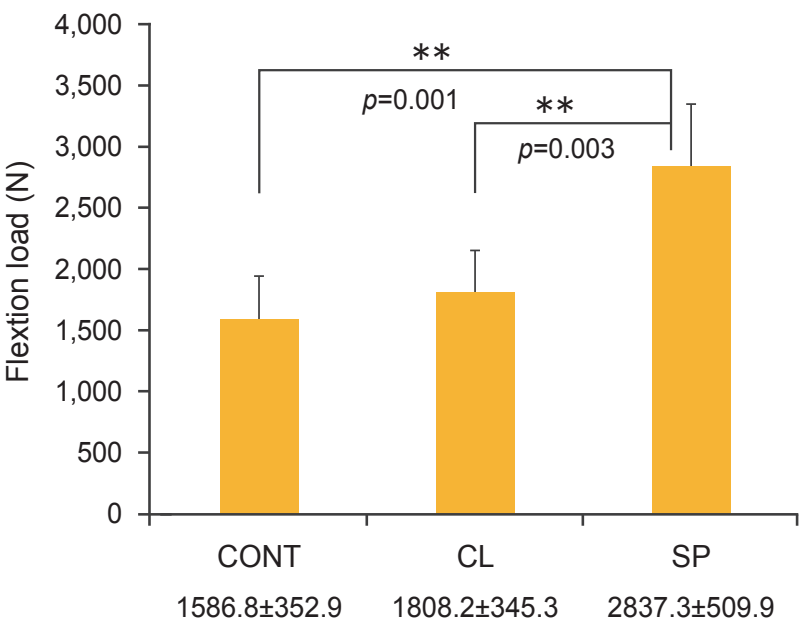

Fig. 6. The improved cross-link (SP) configuration displayed a significantly increased flexion stiffness with respect to the other two configurations tested (CONT, $p=0.001 ; C L, p=0.003$ ). CONT, un-cross-linked controls; $C L$, conventional cross-link; $S P$, cross-link passing through the base of the spinous process. ${ }^{* *} p<0.01$.

$(p=0.17)$ (Table 1, Fig. 6).

\section{Discussion}

The main objective of this study was to test the hypothesis that a novel cross-link configuration with a pair of crosslink devices passing through the base of the spinous processes would increase mechanical resistance against pullout and flexion loads compared with un-cross-link rod only controls or with constructs employing conventional cross-link constructs.

Long rigid fixation from the thoracic to lumbar spine 
Table 1. Load in pullout and flexion testing

\begin{tabular}{lcccc} 
Group & Pullout average (N) & Pullout SD & Flexion average (N) & Flexion SD \\
CONT & 758.7 & 235.9 & $1,586.8$ & 352.9 \\
CL & 924.1 & 168.7 & $1,808.2$ & 345.3 \\
SP & 1146.8 & 345.3 & $2,837.3$ & 509.9 \\
\hline
\end{tabular}

SD, standard deviation; CONT, un-cross-linked controls; CL, conventional cross-link; SP, cross-link passing through the base of the spinous process.

sometimes results in junctional kyphosis and instrument failure. A pedicle screw with rod construct alone cannot provide sufficient strength for thoracolumbar spine injuries, and increasing the number of involved vertebral bodies increases the construct strength. Recovering mechanical properties of the anterior and middle spinal column is valuable for reducing the load sharing of the posterior instrument [14]. Although transverse cross-links add strength to thoracic and thoracolumbar pedicle screwrod constructs, it may be impossible to completely prevent these surgical changes. Sublaminar wires, hooks, and other hook-pedicle screw hybrid constructs are often used at the proximal or distal end of posterior instrumentation as an ancillary means to pedicle screw-rod fixation. However, they also cannot effectively prevent instrument failure. While Dick et al. [2] reported that cross-link devices did not significantly increase axial, flexion-extension, or lateral stiffness of instruments, torsional rigidity significantly increased regardless of the construct, with a pair of crosslinks providing superior resistance to rotary motion compared to that with a single device. The increased torsional stiffness provided by cross-links is most important in long fusions, where torsional loads throughout the entire length of the intervertebral rod can generate cumulative displacements and loss of correction. Pedicle screws provide torsional stability to long thoracic and thoracolumbar constructs comparable to any combination of cross-links [5], but cross-links also resist lateral displacement and quadrilateral shift of paired rods and increase screw pullout strength by linking bilateral instruments [15]. Valdevit et al. [16] showed that under pure torsional loading, a paired diagonal cross-link configuration was the most stable construct when compared with rod only controls or with constructs employing transverse cross-link assemblies resulting in rectangular configurations.

During flexion-compression testing, a partial corpectomy was made in the ventral aspect of the middle vertebral body for applying flexion loads on the instruments and vertebral column. A novel cross-link configuration was the most stable construct, in comparison with rod only controls and with conventional cross-link configurations. However, conventional transverse cross-link configurations were not significantly more stable than rod only controls. These results are in line with the results of the aforementioned report by Dick et al. [2].

Under conditions of sustained flexion-compression, the vertebral column was bent forward, and the intervertebral rods were bent more strongly. In the spinous processes group, the cross-links, which are fixed at the base of the spinous processes, were observed to prevent rod warping. The spinous process played a very important role in preventing vertebral column fracture or instrument failure. During pullout testing, a conventional cross-link increased the pullout strength of the screw-rod system (22\% stronger than controls) by linking bilateral instruments. Furthermore, because the spinous process prevents rod warping, the novel cross-link configuration was $24 \%$ stronger than a conventional cross-link configuration.

Dick et al. [2] and Korovessis et al. [17] reported that the contribution to torsional stiffness by any cross-link device was proportional to the cross-sectional diameter of the cross-link. However, in this study, 2-mm crosslink devices were applied in each construct. This device is relatively thinner, but much easier to operate and can penetrate the base of the spinous process compared to a conventional cross-link device. Before using this thin cross-link device, we were unable to make novel cross-link configurations because a conventional cross-link device is too thick to handle and easily breaks the spinous process. In this study, the thoracic and lumbar spines from each pig were used for each pullout and flexion-compression test. In the thoracic pig specimen, each spinous process stands between the pedicle screw heads on both sides of the same vertebrae. Thus, a conventional cross-link device is too bulky to link bilateral intervertebral rods.

The sample size in this study was relatively small and the pedicle screw insertion angle and length were limited to apply this finding to humans. Mikles et al. [18] 
reported that the height of the pedicle screw construct is an important factor in strengthening a screw-rod system. However, the number of available specimens was limited. To reduce the number of animal specimens, we selected this experimental system using thoracic and lumbar spines from each pig. We also only assessed the immediate postoperative state and did not take any long-term effects of the pedicle screws, such as bony in-growth or degeneration, into consideration. This could be an important factor concerning the pedicle screw pullout and pedicle screwrod system flexion-compression tests. For assessment of screw loosening, repetitive loading in flexion/extension would be required. Therefore, it is not possible to draw any conclusion on long-term outcomes and pedicle screw loosening.

\section{Conclusions}

Under pullout loading in a porcine model, a novel crosslink configuration with cross-link devices passing through the base of the spinous processes provided a stronger construct than un-cross-link rod only controls. In addition, during flexion loading, the improved cross-link configuration was the most resistant to instrument failure, when compared with controls or with constructs employing the conventional transverse cross-link.

\section{Conflict of Interest}

The submitted manuscript does not contain information about medical device(s)/drug(s). Yufu Itonaga Co., Ltd. granted funds in support of this work. Relevant financial activities outside the submitted work include grants, consultancy, and royalties.

\section{Acknowledgments}

The authors thank Dr. Joel Delecrin for his demonstration of the original crosslink configuration (Department of Orthopaedics, Hôtel Dieu Hospital, University of Nantes).

\section{References}

1. Asher M, Carson W, Heinig C, et al. A modular spinal rod linkage system to provide rotational stability. Spine (Phila Pa 1976) 1988;13:272-7.

2. Dick JC, Zdeblick TA, Bartel BD, Kunz DN. Mechan- ical evaluation of cross-link designs in rigid pedicle screw systems. Spine (Phila Pa 1976) 1997;22:370-5.

3. Lynn G, Mukherjee DP, Kruse RN, Sadasivan KK, Albright JA. Mechanical stability of thoracolumbar pedicle screw fixation: the effect of crosslinks. Spine (Phila Pa 1976) 1997;22:1568-72.

4. Pintar FA, Maiman DJ, Yoganandan N, Droese KW, Hollowell JP, Woodard E. Rotational stability of a spinal pedicle screw/rod system. J Spinal Disord 1995; 8:49-55.

5. Wood KB, Wentorf FA, Ogilvie JW, Kim KT. Torsional rigidity of scoliosis constructs. Spine (Phila $\mathrm{Pa}$ 1976) 2000;25:1893-8.

6. Quirno M, Kamerlink JR, Valdevit A, et al. Biomechanical analysis of a disc prosthesis distal to a scoliosis model. Spine (Phila Pa 1976) 2009;34:1470-5.

7. Helgeson MD, Shah SA, Newton PO, et al. Evaluation of proximal junctional kyphosis in adolescent idiopathic scoliosis following pedicle screw, hook, or hybrid instrumentation. Spine (Phila Pa 1976) 2010; 35:177-81.

8. Denis F, Sun EC, Winter RB. Incidence and risk factors for proximal and distal junctional kyphosis following surgical treatment for Scheuermann kyphosis: minimum five-year follow-up. Spine (Phila Pa 1976) 2009;34:E729-34.

9. Jones GA, Kayanja M, Milks R, Lieberman I. Biomechanical characteristics of hybrid hook-screw constructs in short-segment thoracic fixation. Spine (Phila Pa 1976) 2008;33:173-7.

10. Weinstein JN, Spratt KF, Spengler D, Brick C, Reid S. Spinal pedicle fixation: reliability and validity of roentgenogram-based assessment and surgical factors on successful screw placement. Spine (Phila Pa 1976) 1988;13:1012-8.

11. McLain RF, Yerby SA, Moseley TA. Comparative morphometry of L4 vertebrae: comparison of large animal models for the human lumbar spine. Spine (Phila Pa 1976) 2002;27:E200-6.

12. Abshire BB, McLain RF, Valdevit A, Kambic HE. Characteristics of pullout failure in conical and cylindrical pedicle screws after full insertion and backout. Spine J 2001;1:408-14.

13. Hart R, Hettwer W, Liu Q, Prem S. Mechanical stiffness of segmental versus nonsegmental pedicle screw constructs: the effect of cross-links. Spine (Phila Pa 1976) 2006;31:E35-8. 
14. Wang XY, Dai LY, Xu HZ, Chi YL. Biomechanical effect of the extent of vertebral body fracture on the thoracolumbar spine with pedicle screw fixation: an in vitro study. J Clin Neurosci 2008;15:286-90.

15. Benzel EC, Baldwin NG. Crossed-screw fixation of the unstable thoracic and lumbar spine. J Neurosurg 1995;82:11-6.

16. Valdevit A, Kambic HE, McLain RF. Torsional stability of cross-link configurations: a biomechanical analysis. Spine J 2005;5:441-5.
17. Korovessis P, Baikousis A, Deligianni D, Mysirlis Y, Soucacos P. Effectiveness of transfixation and length of instrumentation on titanium and stainless steel transpedicular spine implants. J Spinal Disord 2001; 14:109-17.

18. Mikles MR, Asghar FA, Frankenburg EP, Scott DS, Graziano GP. Biomechanical study of lumbar pedicle screws in a corpectomy model assessing significance of screw height. J Spinal Disord Tech 2004;17:272-6. 